\title{
Design of an Optical Fiber Amplifier with Multiple Serial Pumping for Space Communications
}

\author{
Dirk Giggenbach
German Aerospace Research Establishment (DLR), Oberpfaffenhofen
Institute for Communications Technology
D-82230 Wessling, Germany \\ Proceedings of SPIE, Vol. 3110-2, p. 392
}

Presented at "The $10^{\text {th }}$ Meeting on Optical Engineering in Israel", Jerusalem, $2-6$ March 1997

\begin{abstract}
The feasibility of an optical fiber amplifier as a booster amplifier for transmitter-terminals of free-space coherent laser communication systems has been investigated. To enhance the amplifier's efficiency and reliability in a harsh space environment a new pumping-scheme has been analyzed and demonstrated in an experimental set-up. The amplifier features efficient multiple serial coupling of the pump light into the multimode core of the double-clad fiber by using directional Y-couplers. Special attention has been paid to fiber-geometry, pump light absorption efficiency in the neodymium-doped single-mode core and the attenuation of pump and signal light.
\end{abstract}

Keywords: fiber amplifier, double-clad fiber, optical space communications, multimode Y-coupler

\section{INTRODUCTION}

The German Aerospace Research Establishment investigates the feasibility of coherent optical free space data transmission for links in satellite networks, between space probes, satellites and ground stations [1].

Nd-YAG lasers operating at $1064 \mathrm{~nm}$ are used because of their good overall power efficiency combined with frequency stability, which is necessary for data transmission using phase modulation and coherent heterodyning in the receiver.

Though Nd-YAG lasers with the required output power $(>1 \mathrm{~W})$ are now available, post-amplification in the transmitter is necessary because the integrated optical phase modulators cannot endure the high power levels. The modulators also cause around $5 \mathrm{~dB}$ signal attenuation [2].

Double-clad fibers (DCF) with a Nd-doped inner signal core surrounded by a multimode pump light core are already used as single mode fiber lasers. DCFs can provide a sufficient single-pass gain when pump light intensity is optimized.

In this paper a means for cascaded multiple pump light leading based on directional multimode Y-couplers is introduced, theoretically examined and compared to experimental results. The proposed amplifier setup provides a modular design that enables redundant pump light sources and scaleable output power, both crucial in optical satellite communications.

\section{EXPERIMENTAL SETUP}

\subsection{Structure and properties of the DCF}

Using double-clad fibers for weakly pumped 4-levelsystem fiber amplifiers enables high-power multimode laser diodes as pump-sources, which can easily be launched into the large outer DCF multimode cladding. The used DCF (produced by IPHT, Jena) has a $5 \mu \mathrm{m}$ diameter single-mode signal core situated concentrically inside the $110 \mu \mathrm{m}$ diameter multimode pump light core. The outer cladding consists of silicon providing the required low refraction index. The pump light occasionally crosses the inner core and thereby excites the Nd-ions.

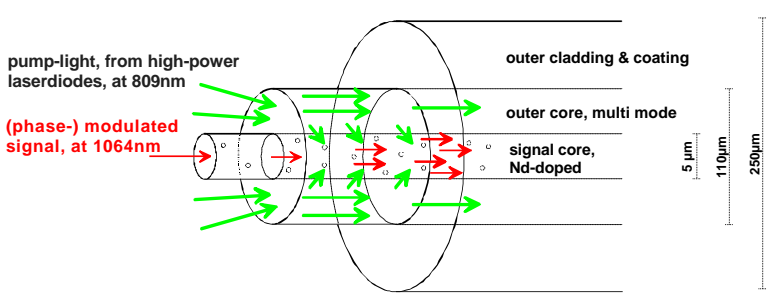

Fig.1 Cross section of the applied double-clad fiber 


\subsection{Pump light leading by Y-couplers}

In a conventional DCF setup the pump light is endcoupled into the multimode core (MMC). This limits the number of pump sources to one laser diode (LD) at each end. By superpositioning two beams through polarizing beam splitter cubes, four LDs can be applied. The LDs are broad area emitters with $\mathrm{P}_{\mathrm{PD}, \text { optical }}=1 \mathrm{~W}$ at $\lambda_{0, \mathrm{p}}=809 \mathrm{~nm}$ which matches well with the absorption bandwidth of the DCF at $804 \mathrm{~nm} \pm 12 \mathrm{~nm}$. For the Y-coupler, the LD-power is first coupled into a multimode coupling fiber (CF) which guides the light into the DCF using a grinded directional multimode $Y$ coupler.

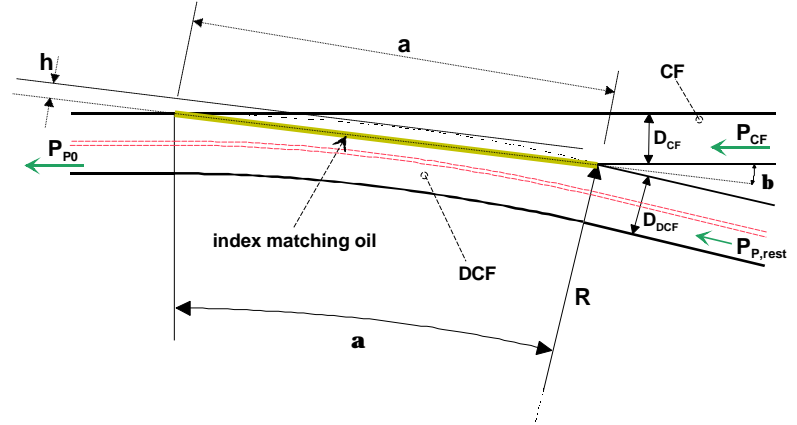

Fig.2 Cut through the multimode $\mathrm{Y}$-coupler

For proper matching, the diameters of both surfaces should be equal. One can solve for the grind-depth "h" and DCF bend-radius "R":

$h=\frac{D_{D C F-M M C}}{2}-\frac{\sqrt{D_{D C F-M M C}{ }^{2}-D_{C F}{ }^{2}}}{2}$

$R=\frac{D_{C F}{ }^{2}}{8 \cdot h \cdot \sin ^{2}(\beta)}+\frac{h}{2}$

The inner core of the DCF is not damaged by this process when $D_{D C F}>D_{C F}$. Using this geometry the axes of both fibers nearly merge into one another.

Index matching oil is needed, otherwise the unavoidable air gap between the two surfaces would cause total inner reflection of the pump light at the lapped fiber end of the CF. Bringing both areas close enough together $(<1 \mu \mathrm{m})$ allows for frustrated total inner reflection, however this was not feasible with standard grinding and positioning equipment. After the alignment process the two parts of the Y-coupler are fixed together and the index matching oil is sealed.

There are two main sources for loss of pump light at the Y-coupler. The small angle of incidence causes reflections at the transitions between the fibers and the index matching oil. To prevent total inner reflection, the relationship $\mathrm{n}_{\mathrm{CF}}<\mathrm{n}_{\mathrm{oil}}<\mathrm{n}_{\text {DCF-MMC }}$ must be observed. At present there is no multimode fiber with exactly the DCF-MMC's refraction index commercially available. The finite angle between CF and DCF causes an optical funnel and leads to radiation losses when steep rays exceed the DCF's maximum angle as shown in fig.3. This can be avoided by using a CF with a smaller numerical aperture (NA) than the DCFMMC, but this lowers the LD-to-CF coupling efficiency.

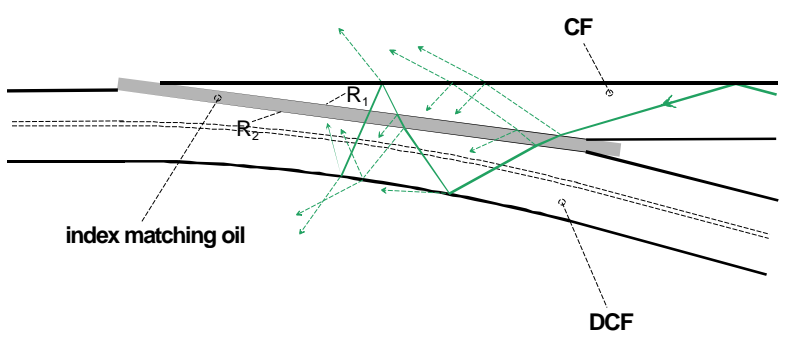

Fig.3 Radiation losses at the Y-coupler

The calculated achievable coupler efficiency is above $80 \%$. Tests with different fibers and index matching oil combinations produced a coupling efficiency $\eta_{Y}$ between $64 \%$ and $86 \%$. With $\mathrm{P}_{\mathrm{PD} \text {,optical }}=1 \mathrm{~W}$, the $\mathrm{Y}$ coupler loss combined with the coupling loss between LD and CF $(\approx 20 \%)$ allow for about $0.7 \mathrm{~W}$ pump power to be introduced into the DCF-MMC.

The attenuation of pump light already inside the DCF before the coupler ("through-coupling attenuation") is approximately $70 \%$.

A way to side-pump the DCF by micro-prisms has been reported by [7] and [12] but with lower coupling efficiency.

\section{SIGNAL AMPLIFICATION MECHANISM}

3.1 Rate equation of the simplified Nd-glass system

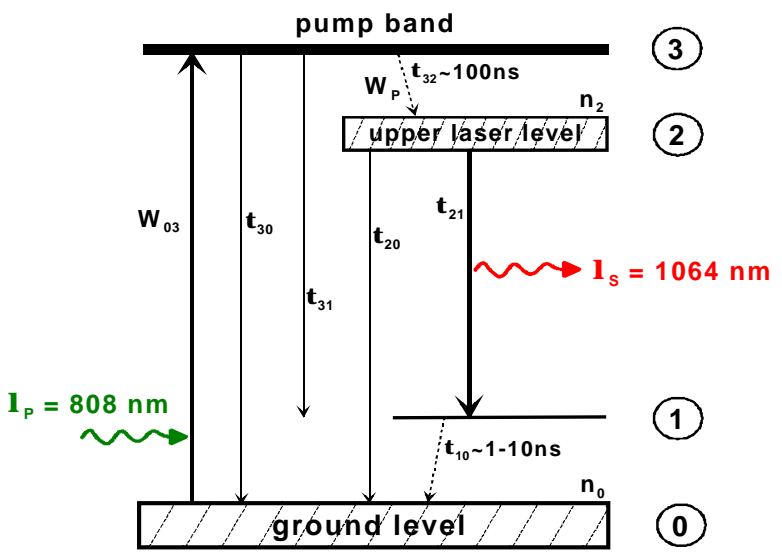

Fig.4 Simplified four level Nd-glass system 
With $\tau_{32}$ and $\tau_{10}$ being very small compared to $\tau_{21}$ the rate equation becomes

$$
\begin{aligned}
& \frac{\delta n_{2}(t, z)}{\delta t}=-\sigma_{21} \cdot c \cdot \phi_{s i g}(t, z) \cdot n_{2}(t, z)- \\
& -\frac{1}{\tau_{F}} \cdot n_{2}(t, z)+\eta_{p} \cdot W_{03}(z) \cdot\left[n_{t o t}-n_{2}(t, z)\right] \stackrel{!}{=} 0
\end{aligned}
$$

The change-rate of level-2 population equals the rate of pumped $\mathrm{Nd}$-ions minus the rate of spontaneous and stimulated emission. In continuous-wave (cw) amplification this rate must be zero.

It will be shown that $n_{2}$ is always small against $n_{\text {tot }}$ in the fiber amplifier so that the pump parameter $\mathrm{W}_{03}$ is not a function of $n_{2}$. The gaussian radial distribution of signal intensity inside the SMC has been approximated by a rectangular distribution so intensity ratios can be substituted with power ratios.

\subsection{Absorption and attenuation of pump light}

The effective absorption coefficient for $808 \mathrm{~nm}$ inside the doped SMC is difficult to calculate due to the uncertain absorption cross section $\sigma_{03}$ but has been measured at $23 \mathrm{~m}^{-1}(100 \mathrm{~dB} / \mathrm{m})$.

The absorption of pump light inside the DCF-SMC depends on the ability of MMC-rays to transit through the SMC. Ideally the pump light power inside the SMC $\left(P_{p, S M C}\right)$ is given by the cross section ratio $\varepsilon_{p, S M C}$

$$
\begin{aligned}
& P_{P, S M C}=\varepsilon_{p, S M C} \cdot P_{P, M M C} \\
& \varepsilon_{p, S M C}=\frac{A_{S M C}}{A_{M M C}}
\end{aligned}
$$

However the mode-selective absorption and reflections distort this simplified image.

The refraction index step between DCF-MMC and -SMC causes small power reflections away from the SMC for steeper angles of incidence, but reaches high values for flat angles of incidence $\left(R=0,25\right.$ for $\varphi=2^{\circ}$ and $R=0,5$ for $\varphi=1^{\circ}$ in our DCF).

As [6] has shown, the absorption coefficient for pump light in a regularly coiled DCF over fiber length starts at a rate approximately twice that of $\varepsilon_{\mathrm{p}, \mathrm{Smc}}$, and after the absorption of the higher order axial modes drops to a value about $1 / 2 \quad \varepsilon_{\mathrm{p}, \mathrm{Smc}}$. Helical modes cannot be absorbed by the SMC. Mode mixing from helical to axial modes can be enhanced by periodically bending the winding of the DCF and by an excentric placement of the SMC or an asymmetric MMC cross section [5]. As the DCF in our experiment is wound up to enhance mode mixing, the cross section ratio is a sufficient approximation and will be applied to further calculations.
The low transparency of the silicon cladding causes an attenuation coefficient of $\alpha_{M M C}=0.007 \mathrm{~m}^{-1}(30 \mathrm{~dB} / \mathrm{km})$ for the total inner reflections at the MMC-silicon transition. The combined absorption and attenuation coefficient for the pump power is

$$
\alpha_{p, t o t}=\alpha_{M M C}+\varepsilon_{p, S M C} \cdot \alpha_{a b s}
$$

\subsection{Amplification and attenuation of signal light}

At $\mathrm{P}_{\text {sig }}=\mathrm{P}_{\text {sat }}$ spontaneous emission equals stimulated emission. $P_{\text {sat }}$ has been calculated through the measured fluorescence lifetime $\tau_{f}=360 \mu$ s to be $6 \mathrm{~mW}$. The inversion-ratio for $P_{\text {sig }} \ll P_{\text {sat }}$ (small signal inversion) can be calculated according to

$$
\frac{n_{2, \text { small }}}{n_{\text {tot }}}=\frac{W_{P} \cdot \tau_{f}}{W_{P} \cdot \tau_{f}+1}
$$

For our amplifier, this ratio could theoretically reach $26 \%$ at coupler locations with $2 \mathrm{~W}$ pump light absorbed optimally and no stimulated emission present. With $\mathrm{P}_{\text {sig }} \gg \mathrm{P}_{\text {sat }}$ (amplifier operating at saturation) this ratio falls to approx. $4 \%$ and thus bleaching of the ground state will not occur. This fact helps us to calculate the pump rate $\mathrm{W}_{\mathrm{p}}(\mathrm{z})$.

$$
W_{p}(z)=\eta_{p} \cdot W_{03}(z)
$$

$\eta_{p}=\left(1+\frac{\tau_{32}}{\tau_{31}}+\frac{\tau_{32}}{\tau_{30}}\right)^{-1} \leq 1$

$W_{03}(z)=\sigma_{03} \cdot c \cdot \phi_{P}(z)$

$\phi_{p}(z)=\varepsilon_{p, S M C} \cdot \frac{P_{p, M M C}(z)}{A_{S M C}\left(h \cdot c \cdot v_{808}\right)}$

The branching ratio $\eta_{p}$ represents losses by pumped Nd-ions that do not end up at the upper laser level. For our fiber $\eta_{p} \approx 0.9$. The small-signal gain $\left(P_{\text {sig }}<<\right.$ $\left.P_{\text {sat }}\right)$ is then

$$
g_{0}(z)=\sigma_{21} \cdot n_{t o t} \cdot \frac{W_{p}(z) \cdot \tau_{f}}{W_{p}(z) \cdot \tau_{f}+1}=\sigma_{21} \cdot n_{2}
$$


and the effective gain with applied signal power

$$
g(z)=\frac{g_{0}(z)}{1+\frac{P_{s i g}(z)}{P_{s a t}}}
$$

The absorption coefficient $\alpha_{\text {sig }}$ caused by the necessary silica dopents is much smaller than in laser crystals, but it becomes a significant design parameter due to the long signal path through the DCF.

The signal power builds up along $z$ according to

$$
\frac{\delta P_{s i g}(z)}{\delta z}=g(z) \cdot P_{s i g}(z)-\alpha_{s i g} \cdot P_{s i g}(z)
$$

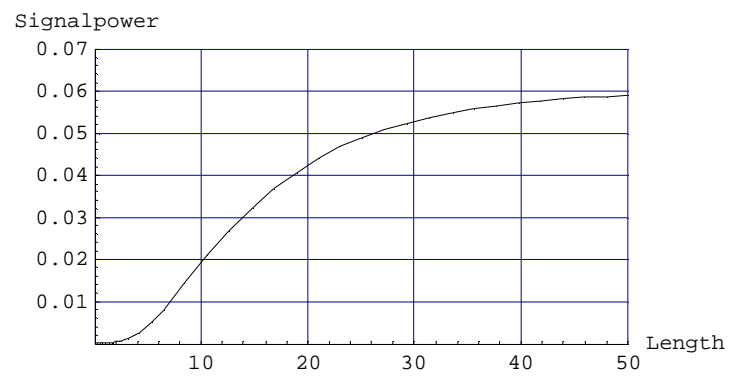

Fig.5 Typical numerical solution of (14) with $\mathrm{P}_{\mathrm{p}}(\mathrm{Z})=$ const. and an initial signal power $\mathrm{P}_{\text {sig } 0} \ll \mathrm{P}_{\text {sat }}$.

Three different gain regions can be distinguished in Fig.5. First $P_{\text {sig }}$ rises exponentially with $g(z) \approx$ constant. When $P_{\text {sig }}>P_{\text {sat }}, g(z)$ starts to fall which causes a net linear growth of $P_{\text {sig. }}$. Finally, the gain equals the attenuation and amplification no longer occurs. In an optimized amplifier setup one can avoid the inefficient first meters before $P_{\text {sig }}>P_{\text {sat }}$ by starting with a signal power above saturation (e.g. with $30 \mathrm{~mW}$ ). This is also important from a communicationtechnological point of view, because a low $\mathrm{P}_{\text {sigo }}$ allows a high amount of amplified spontaneous emission (ASE).

Fig. 5 is not a realistic representation because $P_{p}$ is not constant over $z$ and therefore $W_{p}(z)$ and $g(z)$ vary as described in the following sections.

\section{GAIN WITH DIFFERENT PUMPING SCHEMES}

In a fiber amplifier the development of $\mathrm{W}_{03}$ and thus $\mathrm{g}$ depend on the way the pump power is coupled to the fiber. By varying the location and orientation of the $Y$ couplers, total signal gain and power efficiency can be influenced.

\subsection{Pumping schemes for one DCF section}

\subsubsection{Co-directional pumping}

The pump light power over fiber length for pumping in the signal direction at $\mathrm{z}=0$ with $\mathrm{P}_{\mathrm{p} 0, \mathrm{c}, \mathrm{MMC}}$ is

$$
P_{p, c, M M C}(z)=P_{p 0, c, M M C} \cdot \exp \left(-\alpha_{p, t o t} \cdot z\right)
$$

with $\alpha_{p, \text { tot }}=0.0645 \mathrm{~m}^{-1}$ for our DCF. Numerical solutions are shown in fig. 6 for three different initial signal powers and two different pump powers.

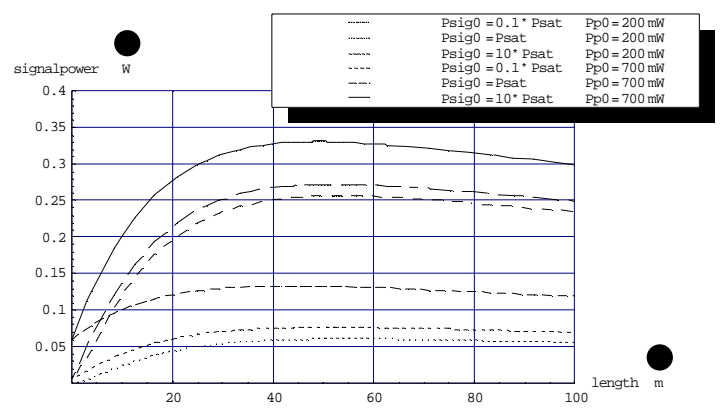

Fig.6 Co-directional pumping with small $\mathrm{P}_{\text {sig0 }}$

In contrast to Fig.5 the signal degenerates when the pump intensity diminishes. With higher initial signal powers the maximum output power is reached after a shorter fiber section. 


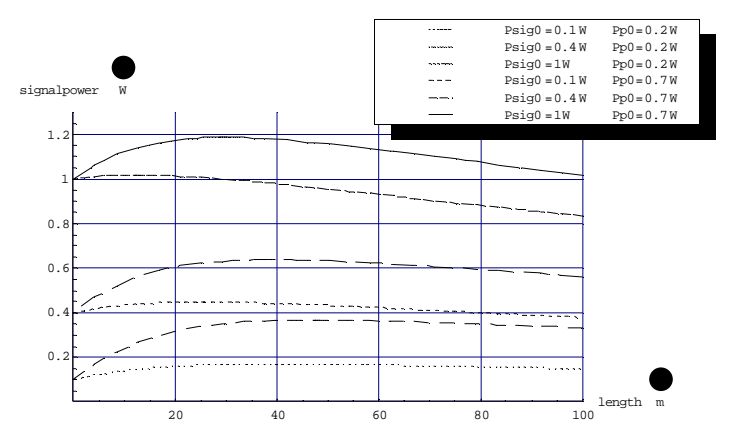

Fig.7 Co-directional pumping with large $\mathrm{P}_{\text {sigo }}$

The gain is decreasing and higher pump power is required to overcome attenuation and obtain useful amplifications.

\subsubsection{Reverse pumping}

In the case of reverse pumping at $\mathrm{Z}=\mathrm{L}$ in the negative z-direction, we derive

$$
P_{p, r, M M C}(z)=P_{p 0, r, M M C} \cdot \exp \left(-\alpha_{p, t o t} \cdot(L-z)\right)
$$

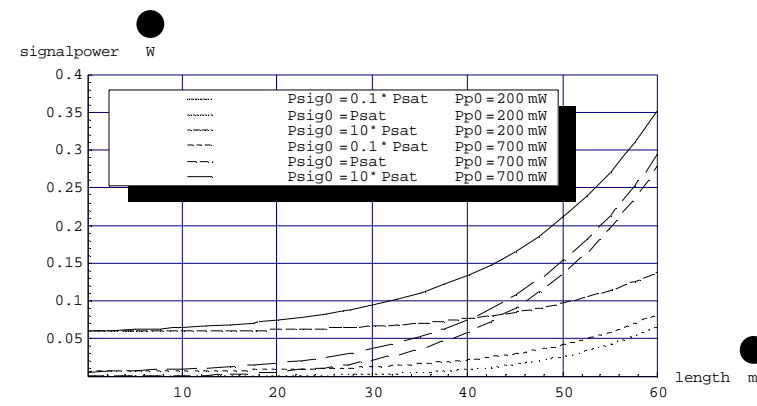

Fig.8 Numerical solutions for reverse pumping at $\mathrm{L}=60 \mathrm{~m}$ with same $\mathrm{P}_{\mathrm{p} 0}$ and $\mathrm{P}_{\text {sigo }}$ as in fig. 6

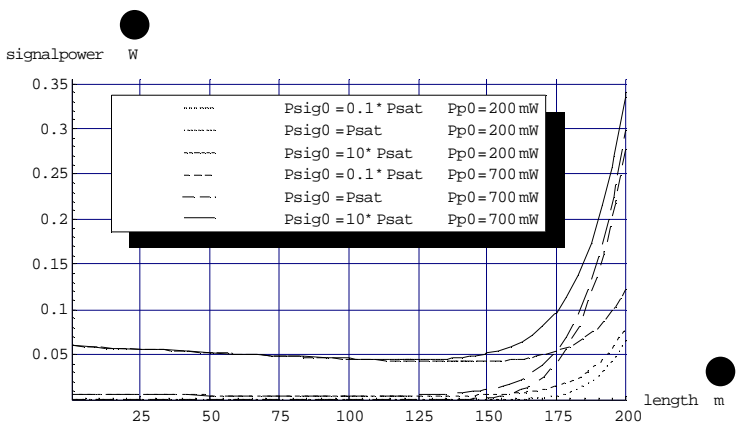

Fig.9 Numerical solutions for reverse pumping with same $P_{p 0}$ and $P_{\text {sigo }}$ as in fig. 6 for $L=200 \mathrm{~m}$ No signal reduction takes place at the end of the section because pump power grows steadily with signal power towards the coupler. But too long a section leads to attenuation at the beginning. However this does not affect signal output very much for small

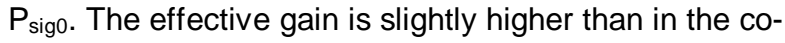
directional pumping case.

With higher initial signal powers, the attenuation due to excessive fiber length rises significantly and the optimum fiber length is shorter.

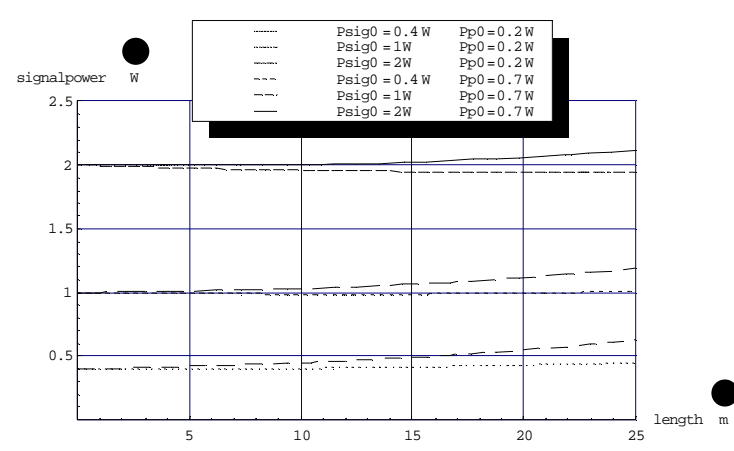

Fig.10 Numerical solutions for reverse pumping with high $\mathrm{P}_{\text {sigo }}$ and optimum $\mathrm{L}$

\subsubsection{Bi-directional pumping}

Pumping from both section ends with $\mathrm{P}_{\mathrm{p} 0, \mathrm{c}}$ at $\mathrm{z}=0$ and $P_{p 0, r}$ at $z=L$ results in the following pump power distribution

$$
\begin{aligned}
& P_{p, b, M M C}(z)=P_{p 0, c, M M C} \cdot \exp \left(-\alpha_{p, t o t} \cdot z\right)+ \\
& +P_{p 0, r, M M C} \cdot \exp \left(-\alpha_{p, t o t} \cdot(L-z)\right)
\end{aligned}
$$

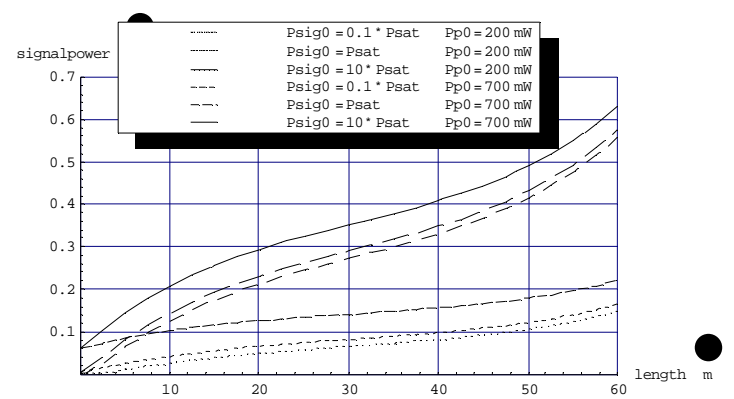

Fig.11 Bi-directional pumping with small $\mathrm{P}_{\text {sig0 }}$ and optimum L 


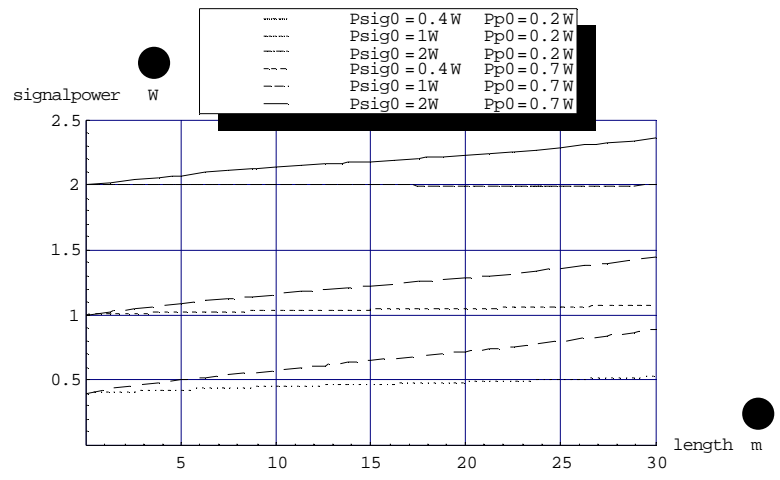

Fig.12 Bi-directional pumping with large $\mathrm{P}_{\text {sigo }}$ and optimum L

\subsubsection{Discussion of the three cases}

Higher pump power causes a better average gain coefficient with high $\mathrm{P}_{\text {sigo }}$ and therefore efficiency rises with stronger LDs.

The optimum fiber length gets shorter as $P_{\text {sigo }}$ increases.

Combinations of co- and reverse-pumping must take into account the optimum fiber length which is derived by the preceding calculations.

In all three cases fiber length is relativly uncritical when $\mathrm{P}_{\text {sig }}$ is small and becomes crucial with higher powers because of the attenuation inside the SMC.

\subsection{Cascaded multiple pumping}

This section discusses signal amplification for one of the several possible pumping schemes.

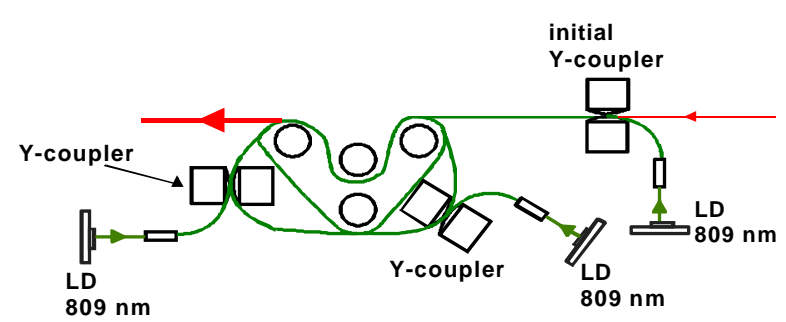

Fig.13 Example for a multiple pumped amplifier constellation. The kidney-shaped winding enhances mode-scrambling.

The investigated setup has an initial reverse-pumping coupler followed by seven bi-directional pumped fiber sections. $P_{\text {sig } 0}=5^{\star} P_{\text {sat }}$ and $P_{p 0}=700 \mathrm{~mW}$ at every $Y$ coupler has been presumed.

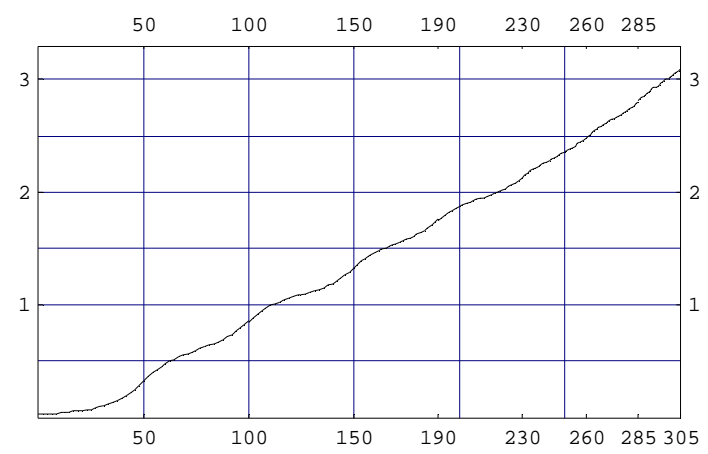

Fig.14 Growing of $\mathrm{P}_{\text {sig }}$ with cascaded bi-directional pumping and optimized fiber section lengths. Couplers are positioned at the marked lengths.

The signal output power after $305 \mathrm{~m}$ of DCF is $3.1 \mathrm{~W}$. This means an efficiency of $3.1 \mathrm{~W} /\left(0.7^{*} 15 \mathrm{~W}\right)=0.30$ inside the DCF.

The remaining pump power from the preceding fiber section is hereby supposed to be totally attenuated inside the Y-Coupler. Through-coupling of this power can be enhanced by optimizing the coupler geometry with this aspect. An arrangement as depicted in Fig.15 would provide the highest gain and efficiency for one fiber section. Methods to enhance through-coupling are discussed in section 6 .

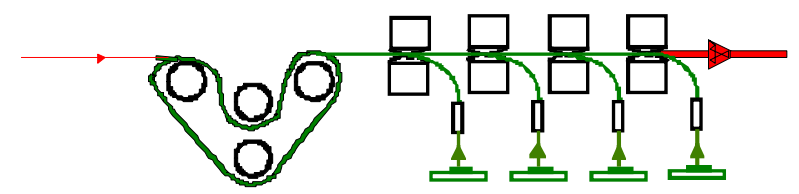

Fig.15 Summing up the powers of several LDs with improved Y-couplers.

\section{RESULTS}

Directional multimode $Y$-couplers have been practically tested to couple up to $86 \%$ of pump light from a standard multimode fiber into a DCF-MMC.

The feasibility of building a cascaded multiple pumped DCF amplifier with output greater than $3 \mathrm{~W}$ has been shown by simulation.

The total calculated system efficiency (socket-to$\mathrm{P}_{\text {sig,out }}$ )

$\eta_{\text {tot }}=\eta_{L D, e l \rightarrow o p t} \cdot \eta_{L D \rightarrow C F} \cdot \eta_{Y} \cdot \eta_{a b s, S M C} \cdot \eta_{808 \rightarrow 1064} \cdot \eta_{p} \cdot \eta_{\text {gain }}$

lies between $7 \%$ and $14 \%$, depending on the applied constellation. The CF-to-output efficiency (without $\eta_{\text {LD,el-opt }}$ and $\left.\eta_{\text {LD-CF }}\right)$ is then between $20 \%$ and $40 \%$.

With higher required output powers the fiber sections between the couplers must be shortened to maintain a high pump rate. This leads to a lower $\eta_{\text {abs,SMc }}$ because remaining pump light inside the DCF is radiated out of 
the $\mathrm{Y}$-coupler. At $\mathrm{P}_{\text {sig }}>12 \mathrm{~W}$ the applicable pump power becomes too weak to maintain a positive gain even with bi-directional pumping and very short sections.

\section{CONCLUSION AND IMPROVEMENTS}

Losses of remaining pump light at the Y-couplers ("through-coupling-efficiency") can be reduced be using a smaller CF as this loss is proportional with the size of the coupler's transitional area. Ignoring the problem of poorer laser diode to CF coupling allows narrower positioning of the couplers and thus enhances $W_{p}$ without losing the pump light from the preceding coupler. Much higher signal output power could then be achieved. This variant has not yet been investigated because our couplers attenuate the remaining pump light too much. However high power laser diodes with improved beam quality are now commercially available, so efficient coupling from the LDs into a pigtailed CF of about $50 \mu \mathrm{m}$ is now feasible.

A computer ray-tracing model of the $\mathrm{Y}$-coupler is being set up to find the optimum coupler geometry. This model will also take into account multiple backreflections along the coupler length, losses by rays that exceed the fibers' N.A., helical modes, polarization dependent effects and losses by non-optimum surfaceshapes and alignment. It would also allow investigations of the mode-distribution and the helicalray ratio inside the DCF-MMC.

The silicon cladding causes problems due to the attenuation of the pump light and its susceptibility to damage. Its thermal instability prevents the construction of a fused Y-coupler between the pump fiber and the DCF. The fiber producer is currently working on a hard-cladding to replace the silicon. A fused coupler combined with matching refraction indices of pump fiber and DCF-MMC would have the lowest coupling losses.

Besides, the DCF can not be glued tightly enough to the polishing block with the soft silicon and so the glass core sometimes breaks during the polishing process, another argument for a hard outer cladding.

Higher Nd-doping will increase the small signal gain coefficient. However from a certain concentration on the Nd-ions tend to build clusters and thus the signal attenuation rises stronger than the overall gain improves. Optimum has to be found.

A smaller MMC to SMC cross-section ratio (maximum possible single mode diameter is approx. $7.5 \mu \mathrm{m}$ ) also increases the pump rate inside the SMC.

Increasing of $\tau_{f}$ could reduce losses by spontaneous emission and thus increase amplifier gain and overall efficiency. The ASE would become smaller and thus the additional signal noise would diminish. In certain Nd-glass, a $\tau_{f}$ of $600 \mu s$ has been observed.
Rayleigh-Scattering has not been regarded as an additional noise source, because after the amplifier fiber there is free space transmission and thus no further scattering takes place like in fiber communications.

Only one variation of several possible coupler constellations could be investigated in this paper as an optimum design depends on too many parameters. A tradeoff must be found between output power and other amplifier requirements, i.e. overall power efficiency, redundancy for LD failures and scaleability of output power.

The concept of multiple cascaded pumping by $\mathrm{Y}$ couplers offers a wide variety of DCF-amplifier setups to fulfill the application-specific differing demands.

\section{ACKNOWLEDGMENTS}

The author wants to thank Prof. Manfred Fickenscher (FH Munich) for helpful discussions and his preceding work on the DCF amplifier and Anton Schex (DLR) for his help with the mathematics.

\section{REFERENCES}

[1] C. Rapp, B. Wandernoth, G. Steudel, A. Schex, DLR Experimental Systems for Free Space Optical Communications, Optical Communications Technology Laser '93, Munich, Germany, June 1993

[2] M. Fickenscher, R. Heilmann, Optische Komponenten für die Weltraumkommuniukation, Z. Flugwiss. Weltraumforsch. 20 (1996) P.18-30, Springer-Verlag 1996

[3] W. Koechner, Solid-State Laser Engineering, Vol. 1, Springer Series in Optical Sciences, $4^{\text {th }}$ Ed., Springer, Berlin, 1996

[4] M. V. Klein, T.E. Furtak, Optik, Springer, Berlin, 1988

[5] A. Tünnermann, H. Zellmer, H. Welling, Faserlaser - Neuartige Laserstrahlquellen mit Emissionen im sichtbaren Spektralbereich, Physikalische Blätter 52 (1996), Nr. 11, p.1123

[6] S. Bedö, W. Lüthy, H. P. Weber, The effective absorption coefficient in double-clad fibers, Optics Communications 1993, p. 331

[7] W. Lüthy, H. P. Weber, High-power monomode fiber lasers, Optical Engineering, August 1995, Vol. 34 No. 8, p. 2361

[8] M. Fickenscher, Efficient Optical Amplifier for Use in Coherent Space Communication, Proceedings of the 
$12^{\text {th }}$ International Congress Laser95 Munich 1995, 324327

[9] Zellmer, et al., High-power cw neodymium-doped fiber laser operating at $9.2 \mathrm{~W}$ with high beam quality, Optics Letters 20 (1995) 578-580

[10] G. J. Ainslie, S. P. Craig, S. T. Davey, The Absorption and Fluorescence Spectra of Rare Earth Ions in Silica-Based Monomode Fibers, J. o. Lightwave Technology, Vol. 6, No. 2, Feb. 1988

[11] J. Stein, Untersuchungen an einem NeodymFaserverstärker zur Satellitenkommunikation, diploma thesis, German Aerospace Research Establishment, 1997

[12] Th. Weber et al., A longitudinal and side-pumped single transverse mode double-clad fiber laser with a special silicone coating, Optics Communications 115 (1995) 99-104

\section{SUMMARY OF DCF PROPERTIES}

\begin{tabular}{|c|c|c|}
\hline$N A_{M M C}$ & 0.38 & 1 \\
\hline$N A_{S M C}$ & 0.14 & 1 \\
\hline $\mathrm{n}_{\mathrm{Nd}}$ & $3 * 10^{25} \quad[1300 p p m]$ & $m^{-3}$ \\
\hline$\tau_{f}$ & 360 & $\mu \mathrm{s}$ \\
\hline$P_{\text {sat }}$ & 0.006 & W \\
\hline$\alpha_{M M C}$ & $0.007 \quad[30 \mathrm{~dB} / \mathrm{km}]$ & $m^{-1}$ \\
\hline$\alpha_{\text {abs }}$ & {$[100 \mathrm{~dB} / \mathrm{m}]$} & $m^{-1}$ \\
\hline$\alpha_{\text {sig }}$ & $0.0028 \quad[12 \mathrm{~dB} / \mathrm{km}]$ & $m^{-1}$ \\
\hline$\sigma_{03}$ & $\approx 1 * 10^{-24}$ & $\mathrm{~m}^{2}$ \\
\hline$\sigma_{21}$ & $\approx 1.1 * 10^{-24}$ & $m^{2}$ \\
\hline $\begin{array}{ll} \\
C\end{array}$ & $1.95 * 10^{8}$ & $\mathrm{~m} / \mathrm{s}$ \\
\hline$v_{1064}$ & $2.8^{*} 10^{14}$ & $\mathrm{~s}^{-1}$ \\
\hline$v_{808}$ & $3.7^{*} 10^{14}$ & $\mathrm{~s}^{-1}$ \\
\hline$\eta_{\mathrm{p}}$ & $\approx 0.9$ & 1 \\
\hline
\end{tabular}

\section{ABBREVIATIONS}

DCF double-clad fiber SMC single mode core MMC multi mode core

LD laser diode

CF coupling fiber, carrying the pump light from the laser diode into the DCF

$\mathrm{W}_{\mathrm{p}} \quad$ pump rate

$\mathrm{W}_{03} \quad$ pump parameter

$\phi_{p} \quad$ photon-flux of pump light inside DCF-SMC

$\phi_{\text {sig }} \quad$ photon-flux of signal light inside DCF-SMC

$\eta_{\mathrm{p}} \quad$ pumping efficiency ("branching ratio")

$\varepsilon_{\mathrm{p}, \mathrm{SMC}}$ transit coefficient for pump light from MMC through SMC

$\alpha_{\text {sig }} \quad$ att. coeff. of signal light in the SMC

$\alpha_{M M C} \quad$ att. coeff. of pump light in the MMC

$\alpha_{\text {abs }} \quad$ absorption coeff. of pump light in the SMC

$\alpha_{\text {tot }} \quad$ combined absorption and attenuation coeff. of pump light

$\sigma_{03}$ absorption cross section of $\mathrm{Nd}$ for $809 \mathrm{~nm}$

$\sigma_{21} \quad$ stimulated emission cross section of Nd for $1064 \mathrm{~nm}$

$\tau_{F} \quad$ fluorescence lifetime without stimulated emission

C speed of light inside the DCF

$P_{\text {sat }} \quad$ saturation signal power

$\mathrm{P}_{\mathrm{p} 0} \quad$ inserted multimode pump power at $\mathrm{Y}$-coupler

$\mathrm{P}_{\text {sigo }} \quad$ signal power at the start of one fiber section

go small signal gain coefficient

g actual signal gain coefficient

ASE amplified spontaneous emission 